\begin{tabular}{|c|c|c|}
\hline Beitr. Ent. & Keltern & ISSN 0005-805X \\
\hline $\mathbf{5 9}(2009) 1$ & S. $1-2$ & 15.07 .2009 \\
\hline
\end{tabular}

\title{
Ehrenpromotion der Ernst-Moritz-Arndt-Universität Greifswald für Bernhard Klausnitzer
}

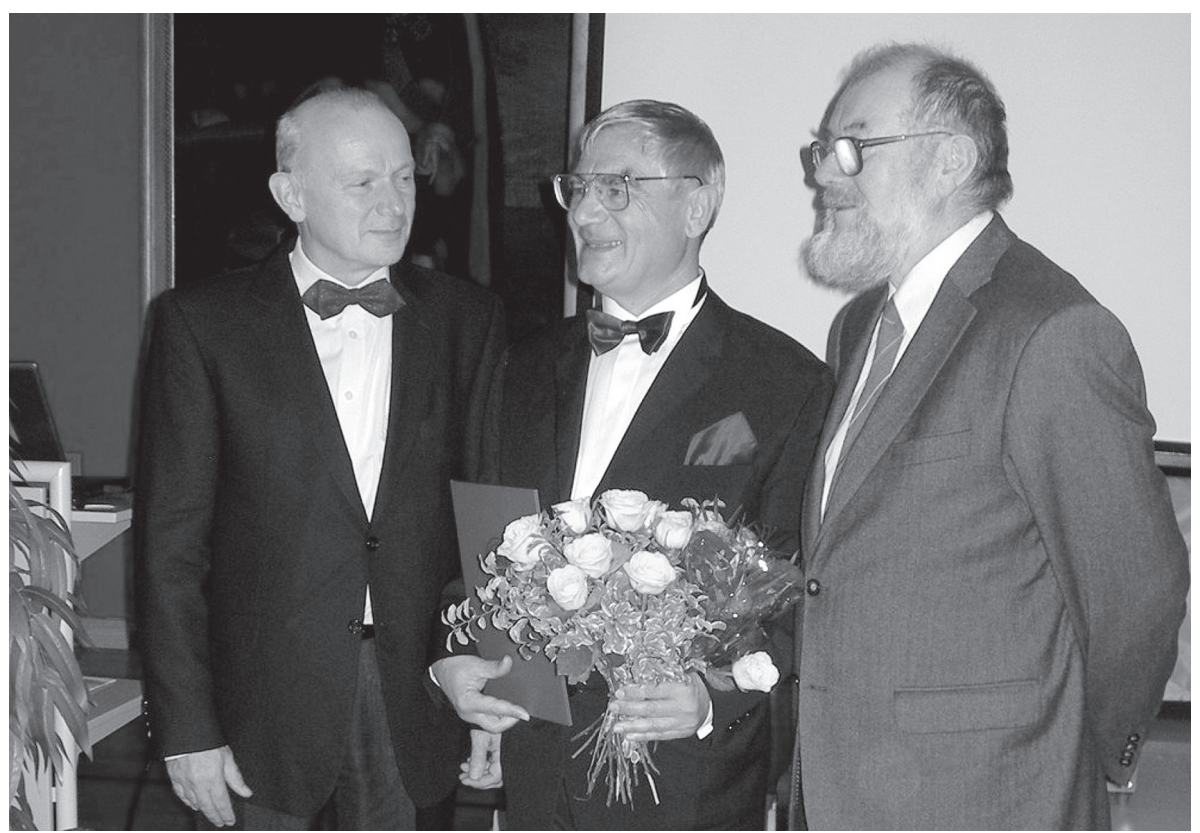

Fig. 1: Prof. Dr. Bernhard Klausnitzer (mitte) mit dem Laudator Prof. Dr. Gerd Müller-Motzfeld (rechts) und dem Festredner Prof. Dr. H. H. Dathe (links). Aufnahme: M. Dannowski.

In einem akademischen Festakt verlieh die Ernst-Moritz-Arndt-Universität Greifswald Herrn Prof. Dr. Bernhard Klausnitzer am 14. November 2008 die Ehrendoktorwürde der MathematischNaturwissenschaftlichen Fakultät. Sie würdigte mit dieser besonderen Ehrung die herausragenden Leistungen von Bernhard Klausnitzer in der entomologischen Forschung und Bildung.

Dekan Prof. Dr. Klaus Fesser überreichte die Urkunde, mit der Prof. Klausnitzer den „Grad und die Würde eines Doktors der Naturwissenschaften honoris causa (Dr. rer. nat. h. c.)“ verliehen wurde. Nach seinen Worten ehrt die Mathematisch-Naturwissenschaftliche Fakultät „den führenden deutschen Entomologen und bedeutendsten europäischen Koleopterologen insbesondere für seine bahnbrechenden Arbeiten zur Larval-Systematik, zur urbanen Ökologie und zur phylogenetischen Systematik, mit denen er auch an der Greifswalder Universität wichtige Forschungsansätze initiierte“. Die Laudatio hielt Prof. Dr. Gerd Müller-Motzfeld, Emeritus der Universität Greifswald. Er war der Antragsteller der Ehrenpromotion, unterstützt vom Direktor des Deutschen Entomologischen Instituts, denn Bernhard Klausnitzer ist seit Jahren gewähltes Mitglied des DEI. Die Laudatio ist im Wortlaut an anderer Stelle veröffentlicht (DGaaE Nachrichten 22 (3): 143-151).

Müller-Motzfeld stellte fest, dass mit Bernhard Klausnitzer ein weltbekannter Entomologe geehrt wird, den er (mit anderen) für den bedeutendsten rezenten Koleopterologen Europas hält. Er 
zähle auch weit über den europäischen Raum hinaus zu den herausragenden Entomologen unserer Zeit. Diese Einschätzung umfasst seinen Kenntnisreichtum, die wissenschaftliche Substanz seiner Arbeiten, die fachliche Vielseitigkeit und sein Engagement für die Entomologie in Wissenschaft, akademischer Lehre und Öffentlichkeit. Dazu schließt es ein umfangreiches Wirken in nationalen und internationalen Fachgesellschaften ein. Seine Aktivität hat auch nach dem Ruhestand 2004 in keiner Weise nachgelassen, so dass diese Ehrung nicht nur Vergangenes anerkennt, sondern auch Ansporn zu künftigen Arbeiten darstellt. Das wissenschaftliche Werk von Bernhard Klausnitzer ist überragend: Sein Schriftenverzeichnis umfasst über 800 Titel, darunter allein 450 wissenschaftliche Original-Arbeiten, 24 Bücher, 26 Kapitel in Fachbüchern sowie Übersetzungen seiner Werke in andere Sprachen, überarbeitete Neuauflagen etc. Der Referent ermittelte über 10.000 Druckseiten Wissenschaft.

Für die Ehrung ist die Ernst-Moritz-Arndt-Universität Greifswald prioritär. An dieser Universität wird die Entomologie seit Carl Gerstäcker (bis 1895 hier Professor der Zoologie) kontinuierlich bis in die Gegenwart vertreten, dies in allen Facetten von der angewandten Entomologie und Parasitologie bis zur Taxonomie, „Arachno-Entomologie“ und zum Naturschutz, wie es kaum sonst in Deutschland der Fall ist. Das Deutsche Entomologische Institut darf sich hier auf jahrzehntelange, sehr enge wissenschaftliche Kooperationsbeziehungen zur Greifswalder Zoologie sowie auf besondere fachliche Traditionen berufen. Herr Professor Müller-Motzfeld war Gründungsmitglied des DEI e. V. und über mehrere Jahre Vorsitzender von dessen Wissenschaftlichem Beirat. Etliche Entomologen des DEI erfuhren in Greifswald ihre Ausbildung, die ihr späteres Wirken prägte (Gerrit Friese, Lothar Zerche, Frank Menzel u. a.). Auch Bernhard Klausnitzers Doktorvater in Dresden, Prof. Dr. Ulrich Sedlag, hatte als ehemaliger Greifswalder wesentlichen Einfluss. Auch er ist dem DEI weiter eng verbunden.

Die Leistungen Bernhard Klausnitzers sind in der jüngsten Vergangenheit im In- und Ausland mehrfach gewürdigt worden, u. a. durch Aspöck (Ent. Basil. 22/2000: 15-19), Naumann (Mitt. DGaaE 13/2001: 7-10) und Dathe (Ent. Nachr. Ber. 43/1999: 149-168, ibid. 48/2004: 153-157). In seiner Laudatio anlässlich der Verleihung der Ehrenmedaille der Societas Internationalis Entomofaunistica Europae Centralis (SIEEC) sagte Professor Aspöck (Wien), Klausnitzer sei „eine der kreativsten, vielseitigsten und produktivsten Persönlichkeiten, die wir heute auf dem Fachgebiet der Entomologie in Deutschland und darüber hinaus haben“"

Eine Bilanzierung des Lebenswerkes von Bernhard Klausnitzer steht jedoch noch lange nicht auf der Tagesordnung, man darf vielmehr mit weiteren profunden Arbeiten rechnen. Gerade in den letzten Jahren sind ausgesprochen reife Werke zum Ergebnis gekommen, die von ihm lange angelegt und zielstrebig verfolgt worden waren. Auch dies sollte mit dieser Ehrung anerkannt und nachdrücklich bestärkt werden.

Redaktion und Wissenschaftlicher Beirat der „Beiträge zur Entomologie“ gratulieren ihrem verdienten Redaktionsmitglied sehr herzlich.

\section{Anschrift des Verfassers:}

Prof. Dr. Holger H. Dathe

Senckenberg Deutsches Entomologisches Institut

Eberswalder Straße 90

15374 Müncheberg

Deutschland 